\title{
High-resolution angle-resolved photoemission spectroscopy of the momentum dependence of the superconducting gap in $\mathrm{Bi}_{2} \mathrm{Sr}_{2} \mathrm{CaCu}_{2} \mathrm{O}_{8}$
}

\author{
T. Yokoya and T. Takahashi \\ Department of Physics, Tohoku University, Sendai 980-77, Japan \\ T. Mochiku and K. Kadowaki \\ National Research Institute for Metals, Sengen, Tsukuba, Ibaraki 305, Japan
}

(Received 22 January 1996)

\begin{abstract}
The momentum dependence of the superconducting gap in $\mathrm{Bi}_{2} \mathrm{Sr}_{2} \mathrm{CaCu}_{2} \mathrm{O}_{8}$ has been studied using highresolution angle-resolved photoemission spectroscopy with nonpolarized light. The size of the superconducting gap obtained at several points on the Fermi surface shows a linear dependence with respect to the gap function of the $d_{x^{2}-y^{2}}$ wave defined as $0.5\left|\cos k_{x} a-\cos k_{y} a\right|$. This suggests that the anomaly in the superconducting gap observed with polarized synchrotron-radiation light is not intrinsic, being probably due to the superstructure of the $\mathrm{BiO}$ plane, and rather the strong anisotropy of the superconducting gap, like the $d_{x^{2}-y^{2}}$ symmetry, is intrinsic. [S0163-1829(96)04822-9]
\end{abstract}

The symmetry of the superconducting order parameter directly relates to the mechanism of superconductivity, in particular, to the pairing force to drive the superconductivity. It is well known that the superconducting order parameter is straightforwardly reflected in the symmetry of the superconducting gap as a function of the wave vector $(k)$ in the Brillouin zone. Angle-resolved photoemission spectroscopy (ARPES) has a unique and powerful capability to determine the electronic structure with respect to the wave vector $(k)$. It is now well established by ARPES that cuprate superconductors have (a) Fermi surface(s) as predicted from the band structure calculation despite the considerable electron correlation among $\mathrm{Cu} 3 d$ electrons. Recent remarkable progress in improving the energy resolution in photoemission spectroscopy ${ }^{1-10}$ enables a precise determination of the size of the superconducting gap and its $k$ dependence.

High-resolution ARPES results on $\mathrm{Bi}_{2} \mathrm{Sr}_{2} \mathrm{CaCu}_{2} \mathrm{O}_{8}$ (Bi2212) have been reported by several groups. ${ }^{1-10}$ Almost all of them show the existence of a large anisotropy of the superconducting energy gap with respect to $k$, indicative of the superconducting order parameter being not a simple $s$ wave. However, the details of the experimental results are not necessarily consistent with each other, leading to conclusions of different symmetries. This discrepancy may arise from the difference in the doping level and/or the quality of samples ${ }^{11}$ and the degradation of the sample surface. ${ }^{5}$ A cooperative effect of the superstructure in the $\mathrm{BiO}$ plane and the strong polarization of the incident light may also complicate the interpretation of ARPES spectra. ${ }^{11}$

In this Brief Report, we report a high-resolution ARPES study on a high-quality Bi2212 single crystal with nonpolarized light. In the course of the study, we paid special attention to checking the existence of an anomaly in the superconducting gap near the $\Gamma X$ line in the Brillouin zone. The $\Gamma X$ direction in the Brillouin zone corresponds to a direction $45^{\circ}$ away from the $\mathrm{Cu}-\mathrm{O}$ bonding in the crystal. Although almost all the ARPES results show a smaller energy gap in the $\Gamma X$ direction than in another high-symmetry direction,
$\Gamma \bar{M}$, parallel to the $\mathrm{Cu}-\mathrm{O}$ bonding, the photoemission spectrum of the $\Gamma X$ line does not necessarily show a definite zero gap, presumably due to the finite energy and angular resolutions, and a major discrepancy in the reported ARPES results lies in this region. Since a small difference in the superconducting gap near the $\Gamma X$ line affects the overall symmetry of the superconducting gap, we expended much care in measuring the superconducting gap near the $\Gamma X$ line as described in the experimental section. We used nonpolarized light to excite photoelectrons in order to reduce the possible cooperative effect of the polarized light and the superstructure in the crystal, which may produce an anomaly near the $\Gamma X$ line. ${ }^{11}$ The result did not show a hump or an extended region of small (or zero) gap near the $\Gamma X$ line, but exhibited a linear dependence of the superconducting gap with respect to the gap function of the $d_{x^{2}-y^{2}}$ wave defined as $0.5\left|\cos k_{x} a-\cos k_{y} a\right|$.

A single crystal of high-quality $\mathrm{Bi} 2212$ was prepared by the traveling solvent floating-zone method using an infrared mirror furnace. Resistivity and Meissner-effect measurements showed superconductivity at $86.4 \mathrm{~K}$ with transition widths of 1.5 and $0.6 \mathrm{~K}$, respectively. This sharp transition indicates a high homogeneity of doping in the crystal. We estimated the composition of the sample using an electronprobe microanalysis; the composition is $\mathrm{Bi}: \mathrm{Sr}: \mathrm{Ca}: \mathrm{Cu}$ $=2.15: 1.86: 0.98: 2.03$ within an error of a few percent. An $\mathrm{x}$-ray rocking-curve analysis has been done for the $(0,0, l)$ reflections and the width of the peak was 150-300 s, which also proves the high quality of the present sample.

Photoemission measurements were performed with a home-built ARPES spectrometer which has a hemispherical analyzer of $300 \mathrm{~mm}$ in diameter. Photoelectrons were excited with nonpolarized light from a discharge lamp (He I;21.22 $\mathrm{eV})$. The base pressure of the spectrometer was about $5 \times 10^{-11}$ Torr. Bi2212 single crystals with different orientations where cleaved in the spectrometer to obtain a fresh and clean surface for photoemission measurements. We did not observe a degradation of the sample surface due to adsorp- 


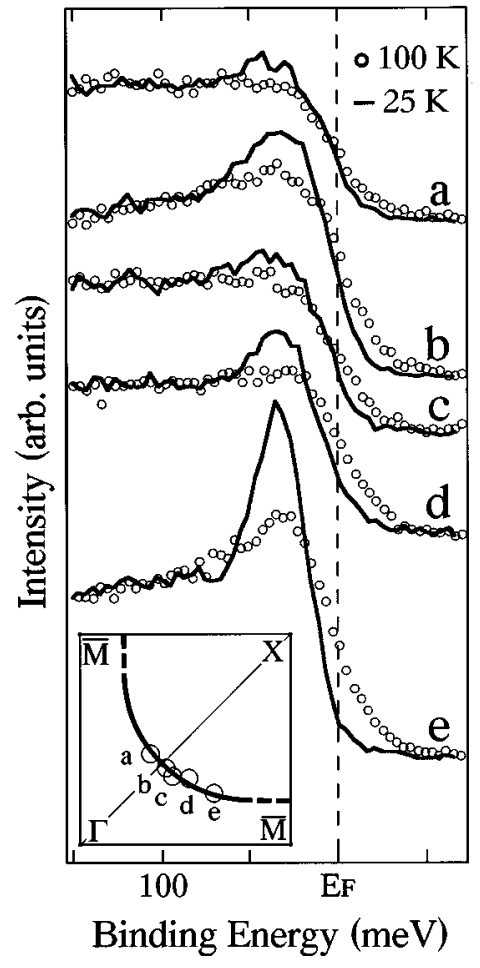

FIG. 1. High-resolution ARPES spectra near $E_{F}$ of a highquality Bi2212 single crystal in the normal $(100 \mathrm{~K}$, circles) and superconducting $(25 \mathrm{~K}$, solid lines) states at five different positions in the Brillouin zone ( $a-e$ in the inset), where a set of normal-state ARPES spectra with $0.5^{\circ}$ interval shows the $E_{F}$ level crossing of the band. The thick solid line connecting these five points in the inset shows a rough sketch of the two-dimensional Fermi surface.

tion of the residual gases even at low temperature during measurement of a few hours. The energy and angular resolution were about $23 \mathrm{meV}$ and $\pm 1^{\circ}$, respectively.

In order to estimate the precise size of the superconducting gap as a function of $k$, it is essential to determine the points of Fermi-level $\left(E_{F}\right)$ crossing in the Brillouin zone (namely, the shape of the Fermi surface) with great accuracy. We measured the normal-state ARPES spectra at $100 \mathrm{~K}$ as a function of polar angle with $0.5^{\circ}$ interval in several direc-

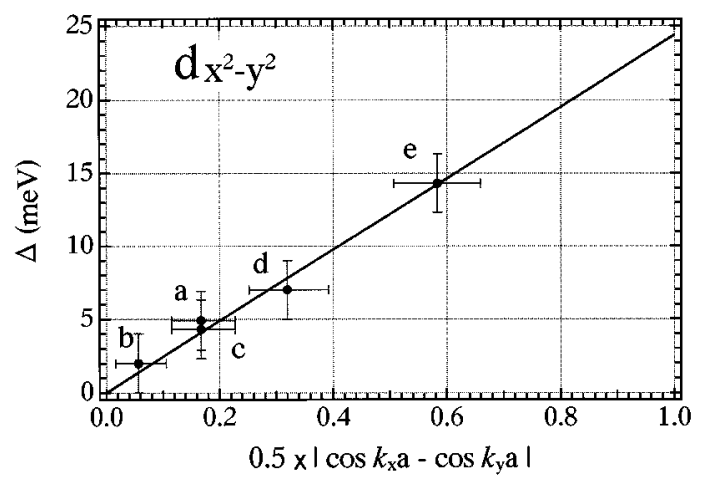

FIG. 2. The observed superconducting gap value $(\Delta)$ plotted with respect to the gap function of the $d_{x^{2}-y^{2}}$ wave defined as $0.5\left|\cos k_{x} a-\cos k_{y} a\right|$. Bars on the experimental points $(a-e)$ show experimental errors due to the finite energy and angular resolutions. Note that the experimental points are on or very close to a straight line which crosses the origin of the coordinate axes.
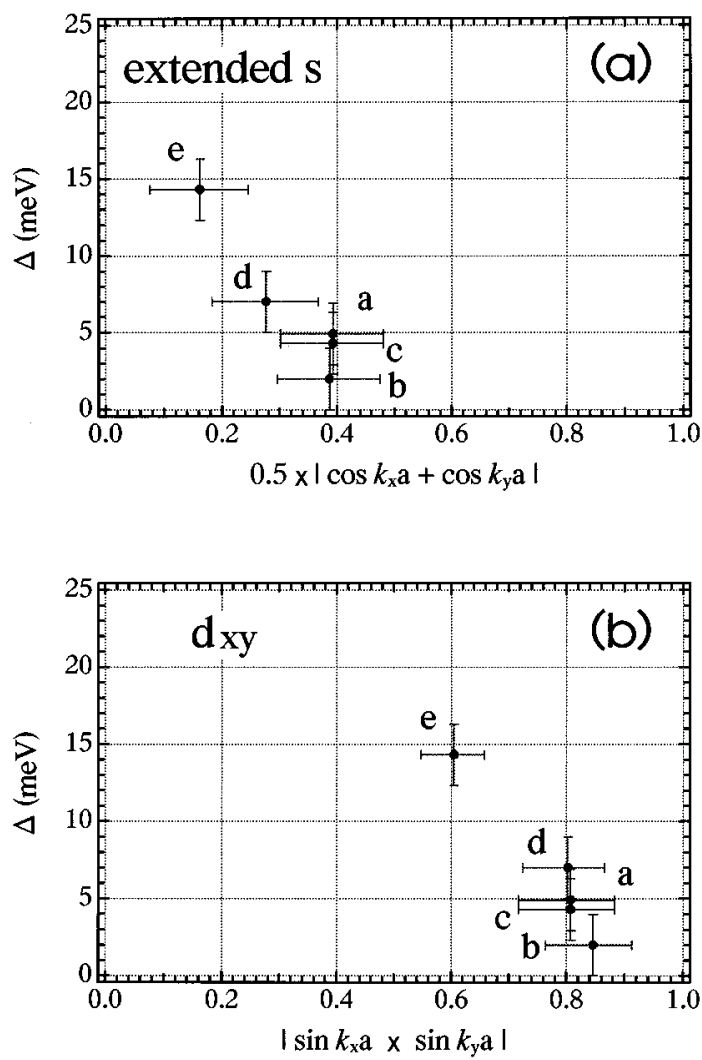

FIG. 3. The observed superconducting gap value $(\Delta)$ plotted with respect to (a) the gap function of an extended $s$ wave defined as $0.5\left|\cos k_{x} a+\cos k_{y} a\right|$ and (b) that of a $d_{x y}$ wave defined as $\left|\sin k_{x} a \sin k_{y} a\right|$. Bars on the experimental points $(a-e)$ show experimental errors due to the finite energy and angular resolutions. In either case, the experimental points are not on a straight line crossing the origin.

tions in the Brillouin zone and found five $E_{F}$ crossing points near the $\Gamma X$ line as shown in Fig. 1. At these five points, we measured photoemission spectra also at $25 \mathrm{~K}$ to estimate the size of the superconducting gap by comparing the normalstate spectra. Following previous studies, we assumed the energy of the middle point of the slope of the photoemission spectra at the $E_{F}$ side to be one-half of the superconducting gap $(\Delta$, where the superconducting gap is $2 \Delta)$, since a photoemission spectrum represents the occupied electronic states. The Fermi level of samples was referenced to that of a gold film deposited on the sample substrate at each temperature and its position was accurate to better than $2 \mathrm{meV}$.

Figure 1 shows the high-resolution ARPES spectra of normal $(100 \mathrm{~K}$, open circles) and superconducting $(25 \mathrm{~K}$, solid lines) states of Bi2212 measured at five different $k$ points in the Brillouin zone (curves $a-e$ ) as shown in the inset. The solid line in the inset connecting these five points shows a rough sketch of the two-dimensional Fermi surface of Bi2212 obtained in this ARPES study, which shows good agreement with previous reports. ${ }^{5-10}$ We find in Fig. 1 that the middle point of the spectral edge in the normal-state spectra is located on or very close to $E_{F}$, indicating that these five measurement points are on or very close to the Fermi surface within the present experimental accuracy. We also find that the spectra at positions $a$ and $c$ look very similar to each other in both the normal and superconducting 
states. This indicates a considerable accuracy in determining the $E_{F}$ crossing points in this study, since, as is seen in the inset of Fig. 1, points $a$ and $c$ are symmetric with respect to the $\Gamma X$ line.

When we cool down the sample from 100 to $25 \mathrm{~K}$, the photoemission spectra exhibit a transfer of spectral weight from the region above $E_{F}$ to that below it (Fig. 1), indicating opening of the superconducting gap. We find that the change is more remarkable in the region away from the $\Gamma X$ line (point $e$ ) than in its vicinity (points $a-c$ ); the spectral edge still stays on or near $E_{F}$ in spectra $a-c$ even at low temperature while in spectrum $e$ the spectral weight at $E_{F}$ is totally transferred to higher binding energy and piles up around 35 $\mathrm{meV}$. This suggests opening of an anisotropic superconducting gap with a larger size away from the $\Gamma X$ line, as is qualitatively consistent with previous reports. ${ }^{4,5,7-9,11}$ In order to study quantitatively the $k$ dependence of the superconducting gap, we assumed the energy of the middle point of the spectral edge in the $20 \mathrm{~K}$ spectrum as the gap value $\Delta$ (the total superconducting gap is $2 \Delta$ ) according to previous studies $^{5,7,9}$ and have plotted it as a gap function of the $d_{x^{2}-y^{2}}$ wave defined as $0.5\left|\cos k_{x} a-\cos k_{y} a\right|$ in Fig. 2. As seen in Fig. 2, we do not observe a hump or a small (or zero) gap region near the $\Gamma X$ line, confirming that the gap anomaly near the $\Gamma X$ line observed with polarized synchrotronradiation light is extrinsic, ${ }^{10}$ being probably due to a cooperative effect of the superstructure in a Bi2212 crystal and the strongly polarized nature of synchrotron-radiation light. ${ }^{11}$ Rather, we find that the experimental points $a-e$ are on or very close to a straight line which crosses the origin of the coordinate axes as shown in Fig. 2.

In order to check the possibility for other symmetries, we also plotted the experimental data as a gap function of the extended $s$ wave defined as $0.5\left|\cos k_{x} a+\cos k_{y} a\right|[$ Fig. 3(a)] or the $d_{x y}$ wave defined as $\left|\sin k_{x} a \sin k_{y} a\right|\left[\right.$ Fig. 3(b)]. ${ }^{12}$ A simple $s$ wave is obviously excluded since the ARPES result shows a strong anisotropy of the superconducting gap. As is clearly seen in Fig. 3, the experimental data are not on a straight line crossing the origin with respect to a gap function of either extended $s$ or $d_{x y}$ symmetry. In contrast, the present experimental data show an almost linear dependence with respect to the gap function of the $d_{x^{2}-y^{2}}$ wave as shown in Fig. 2, suggesting that the $d_{x^{2}-y^{2}}$ symmetry is the most probable superconducting order parameter in Bi2212.

In conclusion, the present high-resolution ARPES study on a Bi2212 single crystal using nonpolarized excitation light has revealed that the anomaly in the superconducting gap near the $\Gamma X$ line observed in an ARPES study using strongly polarized synchrotron-radiation light is not intrinsic, being probably due to a cooperative effect of the superstructure in the $\mathrm{BiO}$ plane and the strongly polarized nature of the excitation light. In contrast, the size of the superconducting gap obtained in this study shows an almost linear dependence with respect to the gap function of the $d_{x^{2}-y^{2}}$ wave, but does not show a consistent result with other symmetries such as extended $s$ or $d_{x y}$, suggesting that the $d_{x^{2}-y^{2}}$ symmetry is the most probable superconducting order parameter in $\mathrm{Bi} 2212$.

We thank H. Matsumoto and T. Koyama for useful discussion. T.Y. thanks the Japan Society for the Promotion of Science (JSPS) for financial support. This work was supported by grants from the Ministry of Education, Science and Culture and the New Energy and Industrial Technology Development Organization (NEDO) of Japan.
${ }^{1}$ C. Olson et al., Science 245, 731 (1989); Solid State Commun. 76, 411 (1990).

${ }^{2}$ R. Manzke et al., Europhys. Lett. 9, 477 (1989).

${ }^{3}$ Y. Hwu et al., Phys. Rev. Lett. 67, 2573 (1991).

${ }^{4}$ B. O. Wells et al., Phys. Rev. B 46, 11830 (1992).

${ }^{5}$ Z.-X. Shen et al., Phys. Rev. Lett. 70, 1553 (1993).

${ }^{6}$ R. J. Kelly, Phys. Rev. Lett. 71, 4051 (1993).

\footnotetext{
${ }^{7}$ R. J. Kelly, Phys. Rev. Lett. 50, 590 (1994).

${ }^{8}$ H. Ding et al., Phys. Rev. B 50, 1333 (1994).

${ }^{9}$ T. Yokoya et al., Phys. Rev. B 51, 3945 (1995).

${ }^{10}$ H. Ding et al., Phys. Rev. Lett. 74, 2784 (1995).

${ }^{11}$ H. Ding et al., Phys. Rev. Lett. 75, 1425 (1995).

${ }^{12}$ M. Sigrist and T. M. Rice, Z. Phys. B 68, 9 (1987).
} 